\title{
An Online Data-Driven Risk Assessment Method for Resilient Distribution Systems
}

\author{
Chaofan LIN, Fei LIU, Liyin ZHANG, Gengfeng LI, Chen CHEN, and Zhaohong BIE
}

\begin{abstract}
Power distribution systems are vulnerable to natural disasters and malicious attacks. An efficient and accurate online risk assessment tool is very necessary to provide timely warning information for emergency dispatch of resilient distribution systems. However, conventional analytical risk assessment methods are subject to known network information, while emerging datadriven methods rarely incorporate resilient resources into the risk assessment procedures, limiting their accuracies when applied to extreme events. To solve the problems, this paper proposes an improved online data-driven risk assessment method adaptive for resilient distribution systems. Twenty-five basic operational indexes from practical experience are chosen to indirectly reflect the system risk, and the complicated relationship between the indexes and risk is characterized by entropy weights and gray correlation degrees. The proposed method is validated on a modified 33-node system, and the results show that it has better accuracy compared with similar approaches in online risk assessment during extreme events. The whole scheme can be helpful for the software design and hardware layout of future resilient distribution systems.
\end{abstract}

Index Terms -Data-driven, entropy weight, gray correlation degree, online risk assessment, resilient distribution system.

\section{INTRODUCTION}

$\mathrm{W}$ ITH modern technology and society relying more and more on continuous electricity supply, power system safety becomes increasingly important. However, the frequent natural disasters these years have been threatening the safe operation of power systems, and the distribution systems are especially affected, due to the complex structure and the vulnerability to extreme environment. For instance, the super typhoon Mangkhut landed in Guangzhou tripped 299 distribution lines, and 440 thousand customers were out

Manuscript received March 14, 2021; revised May 15, 2021; accepted May 20, 2021. Date of publication June 30, 2021; date of current version June 15 , 2021. This work was supported in part by the National Key Natural Science Foundation of China under Grant 51637008, in part by the Research Project of Guangzhou Power Supply Bureau, Guangdong Power Grid Co., Ltd. under Grant 080016KK52180001, and in part by the Fundamental Research Funds for Central Universities under Grant xzy022020028. This paper was presented in part at the 4th International Conference on HVDC (HVDC 2020), Xi'an, China, September 2020. (Corresponding author: Gengfeng Li.)

All authors are with the State Key Laboratory of Electrical Insulation and Power Equipment (Institute of Power System and Its Resilience, Xi'an Jiaotong University), Xi'an 710049, China (e-mail: lin.xjtu@yahoo.com; aaron0520@ stu.xjtu.edu.cn; zhangliyin@stu.xjtu.edu.cn; gengfengli@mail.xjtu.edu.cn; morningchen@mail.xjtu.edu.cn; zhbie@mail.xjtu.edu.cn).

Digital Object Identifier 10.24295/CPSSTPEA.2021.00012 of electricity, with a loss of load of 496.51 MW, in 2018. In this circumstance, it's very necessary to study the online risk assessment method for resilient distribution systems, since it provides early warning information to power supply company that can help system operators make redispatch decisions to mitigate the risk.

Conventional methods used in distribution system risk assessment can be divided into two categories: simulation and analytical methods [1]. The simulation methods, such as sequential [2] and nonsequential [3] Monte Carlo simulation, evaluate the system states sampled by the probability models of system components. While the analytical methods [4], [5] are only based on a limited set of system states with higher probabilities. However, the methods in both categories need known topology and parameter information of a certain distribution system, which is actually changeful due to weather condition, reconfiguration, etc. Besides, the modern distribution systems consist of various uncertainties, such as distributed generators, controllable loads and electric vehicles, which cannot be fully considered in conventional methods.

In recent years, the data-driven risk assessment method has attracted much attention. The contributions of various factors, such as loss of supply, defective equipment, adverse weather, etc. to distribution system risk are analyzed in [6]. Based on fault statistics, the artificial neutral network (ANN) model is adopted in [7] to represent momentary failure rate for risk assessment in terms of the most influential factors. In [8], the big data technology combined with the ANN is utilized for operational reliability (or risk) evaluation, where the data sources are partitioned into four types including accounting, operation, load and other data. To overcome the strict requirement of a large amount of data for training the ANN, an improved logistic regression model is proposed in [9] for operational reliability prediction, considering multiple meteorological factors. The aforesaid studies mainly focus on the normal conditions of distribution system operation, and the methods cannot be used for the risk assessment of resilient distribution systems under extreme events, because many factors like resilient resources and emergency response capabilities are not incorporated in. With more simplified training models, the analytic hierarchy process (AHP) and fuzzy comprehensive evaluation (FCE) have been widely adopted to determine the preference information [10]-[13] Specifically, [14] proposes a group AHP based method to assess the lightning vulnerability of distribution networks and 
provides a quantitative reference for network planning under natural disasters. However, the objective information may not be fully used in the AHP and FCE which would decrease the risk assessment result.

To consider more on extreme events, [15] designs a disastermitigation dispatch platform based on big data mining of disaster prevention and emergency response information, which can be used for decision-making of resilient distribution systems. Specifically, a risk-based security assessment method is proposed in [16] for wet snow extreme events, using the big data from weather, grid, and renewable energy resource (RES). However, the key features of resilient distribution systems have not been well integrated, and a general scheme for datadriven risk assessment program design has not been established. Based on the practical experience from local power supply company, our previous work in [17] provides a novel datadriven approach for the real-time risk assessment of resilient distribution systems during typhoon weather, which is extended in this paper. The main contributions are listed as below:

1) A practical online data-driven risk assessment scheme is established for applications in resilient distribution systems.

2) A real-time measurement database from practice is constructed as the input of risk assessment, and the data of areabased resilience metric is taken as the benchmark of risk.

3) The entropy weight and gray correlation degree are combined to characterize the complicated relationship between the risk and the measured data accurately and efficiently.

The rest of the paper is organized as follows: Section II introduces the risk assessment database of 25 indexes as well as their access ways, and Section III presents the area-based resilience quantification method. The combined entropy weight and gray correlation method for data mining of the indexes and risk are elaborated in Section IV, and the scheme for real application is illustrated in Section V. The case study to a modified 33-node test system is conducted in Section VI, and the conclusion is drawn in Section VII.

\section{BAsic InDEXES FoR OnLINE Risk Assessment}

To conduct the online risk assessment of distribution systems during extreme events, the first step is to screen out the key risk indicators. According to the International Strategy for Disaster Reduction, risk consists of hazard and vulnerability [18]:

$$
\text { Risk }=\text { Hazard } \times \text { Vulnerability }
$$

In this paper, a hierachical index system for risk assessment is constructed. This index system divides the risk indicators of resilient distribution systems from the aspects of electric load, power equipment and network, resilient resources, emergency management, and geological and meteorological conditions. According to the practical demand of Guangzhou Power Supply Bureau and the feasibility in online risk assessment, 25 resilient indicators in 5 categories in Table I are selected as the basic indexes for risk assessment.

The real-time data of the 25 indexes in Table I can be obtained from various sources in real applications. The
TABLE I

INDEXES FOR ONLINE RISK ASSESSMENT

\begin{tabular}{lcc}
\hline \hline No. & Category & Index \\
\hline 1 & Loss of load probability (LOLP) \\
2 & Load & Expected duration of loss of load (EDLL) \\
3 & Expected ratio of system loss of load (ERSLL) \\
4 & & Expected ratio of maximum loss of load (ERMLL) \\
5 & Ratio of current loss of load (RCLL) \\
\hline 6 & Average load rate of lines (ALRL) \\
7 & Grid & Maximum load rate of lines (MLRL) \\
8 & & Average load rate of transformers (ALRT) \\
9 & & Maximum load rate of transformers (MLRT) \\
\hline 10 & & Photovoltaic power generation forecast (PPGF) \\
11 & & Wind power generation forecast (WPGF) \\
12 & & Online capacity of energy storage devices (OCESD) \\
13 & Resilient & SOC of energy storage devices (SOCESD) \\
14 & resources & Capacity of controllable load (CCL) \\
15 & & Response speed of controllable load (RSCL) \\
16 & & Online capacity of diesel generators (OCDG) \\
17 & & Forecast available time of diesel generators (FATDG) \\
\hline 18 & & Number of mobile power-generating vehicles (NMPGV) \\
19 & Emergency & Forecast available time of mobile power-generating \\
20 & response and & vehicles (FATMPGV) \\
21 & repair & Number of repair crew members (NRCM) \\
22 & & Number of repair supplies (NRS) \\
23 & & Efficiency of repair sources in place (ERSP) \\
\hline 24 & Meteorology & Warning level of meteorological disaster (WLMD) \\
25 & Real-time weather severity (RTWS) \\
\hline \hline & &
\end{tabular}

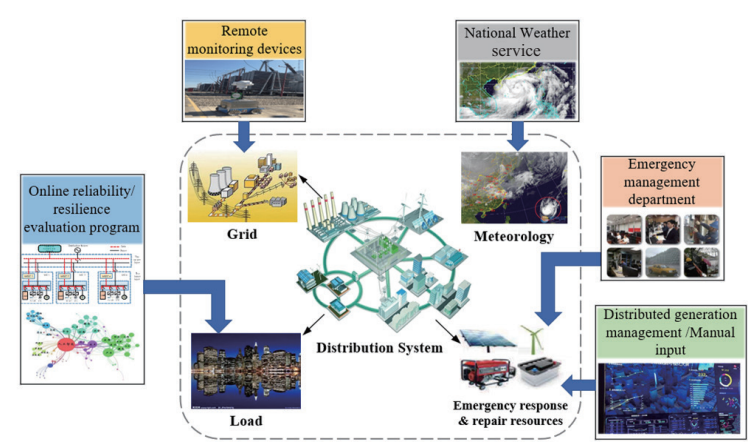

Fig. 1. Real-time data sources and collection.

expected load information is calculated by online reliability evaluation program. The current load and grid information is obtained from remote monitoring devices. The resilient resources information is provided and forecasted by distributed generation management system or inputted manually. The emergency response and repair resource information are provided by emergency management department of distribution system operators. And finally, the meteorological information is obtained from National Weather Service. The various real-time data sources and their integration are shown in Fig. 1.

For those indexes that may change at any time, it is necessary to ensure the real-time performance of the proposed risk assessment method. For instance, for index ERSP(22), the wireless communication can be used to locate the distribution 


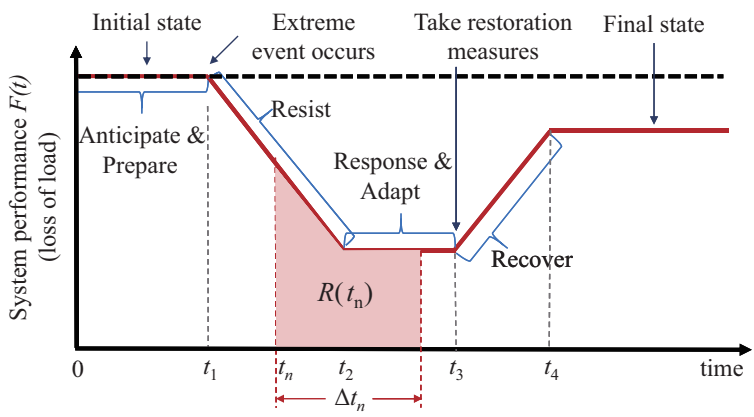

Fig. 2. Typical system performance curve during an extreme event.

of maintenance personnel and materials and confirm their conditions. For index $\operatorname{ICDQ}(23)$, the critical nodes and lines list obtained from power grid company can be combined with the damaged equipment to ensure the real-time performance.

\section{ArEA-BASED RESILIENCE QUANTIFICATION}

The resilience of distribution systems can be quantified by energy not supplied during a certain period [19]. A typical system performance curve is shown in Fig. 2.

A resilient distribution system usually undergoes four phases for an extreme event, including the anticipation and preparation, resistance, response and adaption, and recovery phases, as are indicated in Fig. 2. In phase I $\left(0-t_{1}\right)$, the system takes necessary preparations such as resilient resources pre-location and component reinforcement so as to increase the robustness in extreme environment. In phases II $\left(t_{1}-t_{2}\right)$ and III $\left(t_{2}-t_{3}\right)$ after the event occurs, emergency responses are triggered and loads start to be cut due to system damages. Resilient resources like distributed generators and energy storage systems are utilized to support some critical loads. In phase IV $\left(t_{3}-t_{4}\right)$ when the extreme event has passed, the system takes restoration measures and the load loss starts to shrink. Repair crews are dispatched to fix damaged components, and along with dynamic changes of system topology, the resilient resources are also coordinated to restore as many loads as possible.

Suppose the current observation time is $t_{n}$ and the resilience quantification period is $\Delta t_{n}$, the standardized area of energy supplied is deemed as the percent value of system resilience:

$$
R_{\mathrm{s}}\left(t_{n}\right)=\frac{100}{\Delta t} \int_{t_{n}}^{t_{n}+\Delta t} F(t) \mathrm{d} t(\%),
$$

where $R_{\mathrm{s}}\left(t_{n}\right)$ is resilience at $t_{n}$, and $F(t)$ is the ratio of actual supplied load to all load, both of which are time-varying.

\section{Data Training With Gray Correlation}

In this section, we will standardize the indexes in Table I and present a comprehensive data-driven risk assessment method based on entropy weight and gray correlation degree.

\section{A. Data Standardization}

The indexes of risk need to be standardized to effectively
TABLE II

Data StANDARdizaTion Formulas

\begin{tabular}{|c|c|c|c|}
\hline No. & Index & Standardization method & Formula \\
\hline $\begin{array}{l}2 \\
17 \\
19\end{array}$ & $\begin{array}{c}\text { EDLL } \\
\text { FATDG } \\
\text { FATMPGV }\end{array}$ & $\begin{array}{l}\text { Use the exponential transfer function } \\
\text { to standardize }\end{array}$ & $U_{i}=1-e^{-S_{i}}$ \\
\hline $\begin{array}{l}10 \\
11 \\
12 \\
16 \\
\end{array}$ & $\begin{array}{c}\text { PPGF } \\
\text { WPGF } \\
\text { OCESD } \\
\text { OCDG }\end{array}$ & Base on the total installed capacity & $U_{i}=S_{i} / S_{\text {Total }}$ \\
\hline 14 & $\mathrm{CCL}$ & Base on the maximum load value & $U_{i}=S_{i} / \operatorname{Load}_{\max }$ \\
\hline 15 & RSCL & $\begin{array}{l}\text { Base on the maximum load response } \\
\text { speed measured by load control center }\end{array}$ & $U_{i}=S_{i} / S_{i, \max }$ \\
\hline $\begin{array}{l}18 \\
20 \\
21\end{array}$ & $\begin{array}{c}\text { NMPGV } \\
\text { NRCM } \\
\text { NRS }\end{array}$ & $\begin{array}{c}\text { Base on the maximum number under } \\
\text { normal circumstances }\end{array}$ & $\begin{array}{c}U_{i}=S_{i} / \\
\text { Number }_{\max }\end{array}$ \\
\hline
\end{tabular}

TABLE III

Data STANDARDiZation For QualtTATIVE INDEXes

\begin{tabular}{lcccccc}
\hline \hline \multirow{2}{*}{ No. } & \multirow{2}{*}{ Index } & \multicolumn{5}{c}{ Scale } \\
\cline { 3 - 6 } & & 0 & 0.25 & 0.5 & 0.75 & 1 \\
\hline 22 & ERSP & Excellent & $\begin{array}{c}\text { Good } \\
\text { Slightly }\end{array}$ & Normal & Poor & Bad \\
23 & ICDQ & Unimportant & $\begin{array}{c}\text { mattered } \\
\text { Bormal Important }\end{array}$ & Critical \\
24 & WLMD & None & Blue & Yellow & Orange & Red \\
25 & RTWS & Normal & Poor & Bad & Damaging & Fatal \\
\hline \hline
\end{tabular}

eliminate the incommensurability, and then the accuracy of the impact of these indexes on distribution system risk assessment can be guaranteed. For indexes LOLP(1), ERSLL(3), ERMLL (4), RCLL(5), ALRL(6), MLRL(7), ALRT (8), MLRT(9), it is not necessary to standardize them since their values are already limited in $[0,1]$. The standardization formulas for other indexes are shown in Table II. For the first type of indexes in Table II, an exponential transfer function can be used to standardize them since their values range from 0 to infinity. For the second, third and fourth types of indexes in Table II, the maximum values of various indexes (such as the maximum total power output of resilient resources and the maximum load value at certain time instant) can be used as benchmarks to standardize the indexes.

For those indexes that cannot be directly quantified, including $\operatorname{ERSP}(22), \operatorname{ICDQ}(23), \operatorname{WLMD}(24)$, and RTWS(25), the standardization can be conducted according to Table III.

\section{B. Weights Determination by Entropy}

The indexes selected for risk assessment of distribution systems during extreme events have different degrees of impact on assessment results. To conduct online risk assessment more accurately, it is necessary to assign the weight of each index to determine the relative importance of the index in risk assessment. In this subsection, the information entropy theory is introduced for weights determination.

The entropy measures the degree of system disorganization. 
In information theory, an increasing entropy indicates a loss of information. An ordered system tends to have a small entropy value and contains a great amount of information; conversely, a system with disorder has a large entropy value and includes little information. Entropy is an ideal scale when applied to the evaluation of different decision-making processes [20]. According to the concept of entropy, the amount and quality of information that an index can provide indicate the significance of the index to the risk assessment.

In the assessment problem with $m$ objects and $n$ indexes, the entropy of the $i$-th index is denoted by $H_{i}$ :

$$
H_{i}=-k \sum_{j=1}^{m} f_{i j} \ln f_{i j}, i=1,2, \ldots, n,
$$

where

$$
f_{i j}=r_{i j} / \sum_{j=1}^{m} r_{i j} ; k=1 / \ln m ; H_{i} \geqslant 0 ; k \geqslant 0 .
$$

The entropy weight of the $i$-th indicator is denoted by $w_{i}$ :

$$
w_{i}=\left(1-H_{i}\right) /\left(n-\sum_{i=1}^{n} H_{i}\right)
$$

Set $f_{i j} \ln f_{i j}=0$ when $r_{i j}=0$.

When the values of a certain index are exactly the same under different situations, the entropy value reaches the maximum of 1 and the entropy weight is 0 , which also means that the index does not provide any useful information for decision making. An index that shows great difference under various conditions has small entropy value and high entropy weight which indicates its significance in providing useful information for decision makers [21].

According to the above formulas, the entropy weight of each index in Table I can be determined. The larger the entropy weight is, the greater the influence of the index is in online risk assessment of resilient distributed systems.

\section{Risk Quantification by Gray Correlation Degree}

A distribution system can be regarded as a gray system because it is difficult to fully characterize the real-time operating status during extreme events. In a gray system, part of information is known and part is not. The gray correlation is the uncertainty correlation between systems or the main factors of systems. The gray correlation analysis (GCA) is a quantitative method to analyze the degree of gray correlation between the various factors in a gray system [22]. Thus, the GCA can be applied to the online risk assessment using the historical data of resilient distribution systems.

Denote the gray correlation factor set by the data $X=\left\{x_{i} \mid i\right.$ $=1,2, \ldots, m\}$ of $m$ objects and $n$ indexes. Regard $x_{0} \in X$ as the mother sequence, $x_{i} \in X$ as the sequence, and $x_{i}(k)(k=$ $1,2, \ldots, n)$ as the $k$-th index of object $x_{i}$. The gray correlation degree of $x_{i}(k)$ to $x_{0}(k)$ can be calculated by:

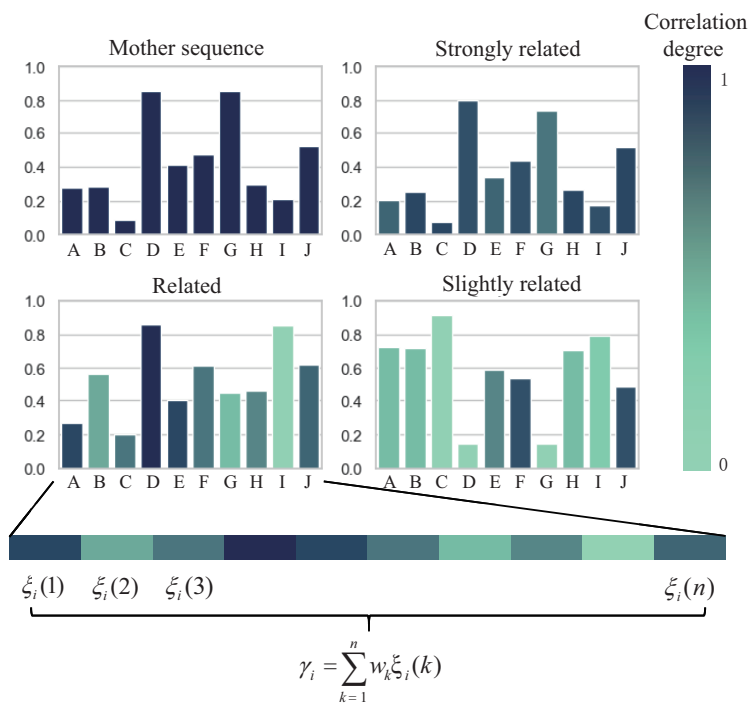

Fig. 3. Gray correlation analysis of 10 basic indexes.

$\xi\left[x_{0}(k), x_{i}(k)\right]=\frac{\Delta \min +\rho \Delta \max }{\Delta_{i}(k)+\rho \Delta \max }, i=1,2, \ldots, m ; k=1,2, \ldots, n$

where $\rho$ is the resolution coefficient with a value in $[0,1]$, and

$$
\begin{gathered}
\Delta_{i}(k)=\left|x_{0}(k)-x_{i}(k)\right|, \\
\Delta \min =\min _{i}\left[\min _{k}\left(\left|x_{0}(k)-x_{i}(k)\right|\right)\right], \\
\Delta \max =\max _{i}\left[\max _{k}\left(\left|x_{0}(k)-x_{i}(k)\right|\right)\right] .
\end{gathered}
$$

When $\xi\left(x_{0}(k), x_{i}(k)\right)$ results in a real number, calculate the correlation degree of $x_{i}$ to $x_{0}$ by:

$$
r\left(x_{0}, x_{i}\right)=\frac{1}{n} \sum_{k=1}^{n} \xi\left[x_{0}(k), x_{i}(k)\right] .
$$

It is noteworthy that the value of resolution coefficient $\rho$ can influence the ranking of gray correlation degrees and should be selected properly. Fig. 3 demonstrates the typical results of the GCA regarding 10 of 25 indexes.

As for the application of GCA in the risk assessment of distribution systems during extreme events, first train the model with historical sample data according to the operation standard of the distribution system and then assess the risk through the gray correlation degree between the real-time and ideal status. The higher gray correlation degree between the real-time and ideal status means that the state tends to be safer. Finally, the gray correlation degrees are transformed to risk values with a pre-defined map and the warning information is prepared with the risk assessment results.

\section{Procedures of the Proposed Method}

The flow chart of the proposed online risk assessment procedures is shown in Fig. 4, and the details are presented as the following steps: 


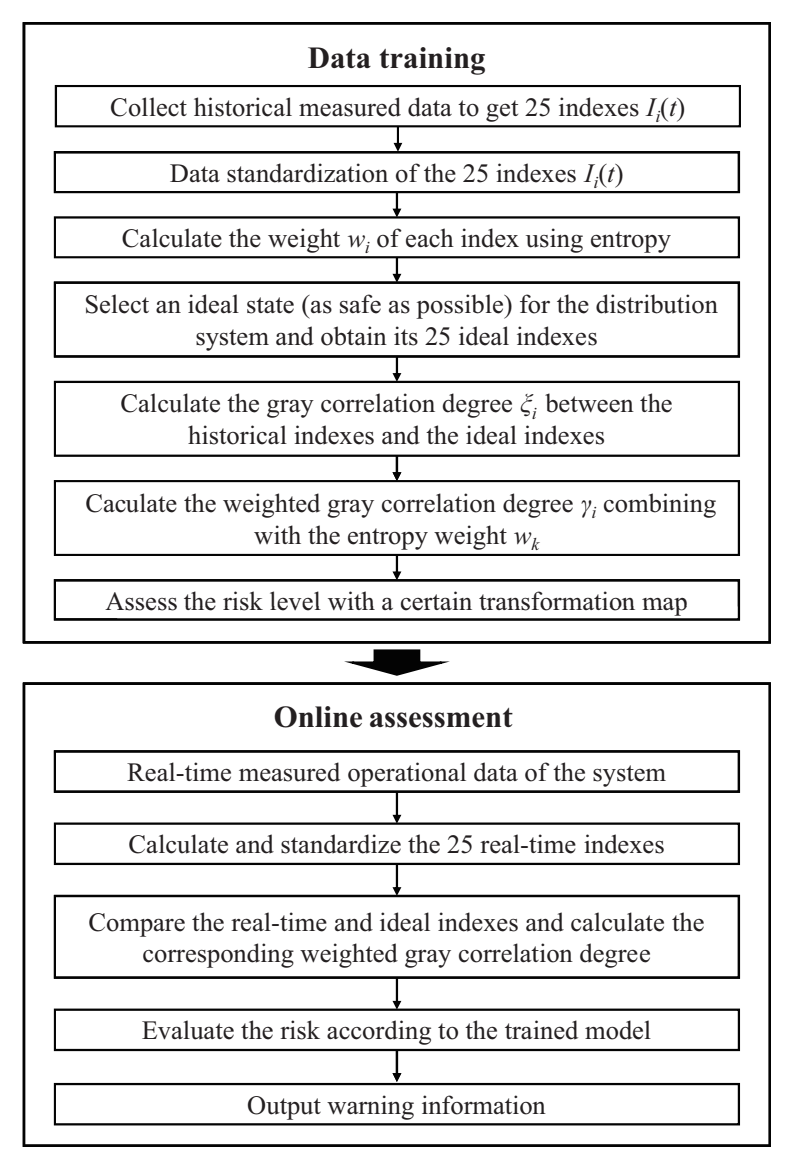

Fig. 4. Flow chart of the proposed method.

1) Obtain the historical data of a distribution system under $m$ different conditions in extreme events. The data under each condition include $n$ risk indexes.

2) Standardize the data according to the formulas in Tables II and III. Denote the data after standardization by $X=\left(x_{i} \mid i=1\right.$, $2, \ldots, m)$ where $x_{i}=\left[x_{i}(1), x_{i}(2), \ldots, x_{i}(n)\right]$.

3) Calculate the entropy weight of each index according to the method in Section III-B.

4) Set $x_{0}$ as the data of an ideal state. Calculate the gray correlation degree between $x_{0}$ and $x_{i}(i=1,2, \ldots, m)$. Denote the result by $\left(\xi_{1}, \xi_{2}, \ldots, \xi_{m}\right)$.

5) Calculate the weighted gray correlation degree between the historical states and ideal state:

$$
\gamma_{i}=\sum_{k=1}^{n} w_{k} \xi_{i}(k) .
$$

6) Assess the risk level with a certain transformation map.

7) Collect the real-time measured data of the system and calculate the corresponding standardized basic indexes.

8) Calculate the weighted gray correlation degree between the real-time state and the ideal state.

9) Calculate the risk values and output warning information.

\section{CASE Study}

The whole proposed risk assessment scheme is applied

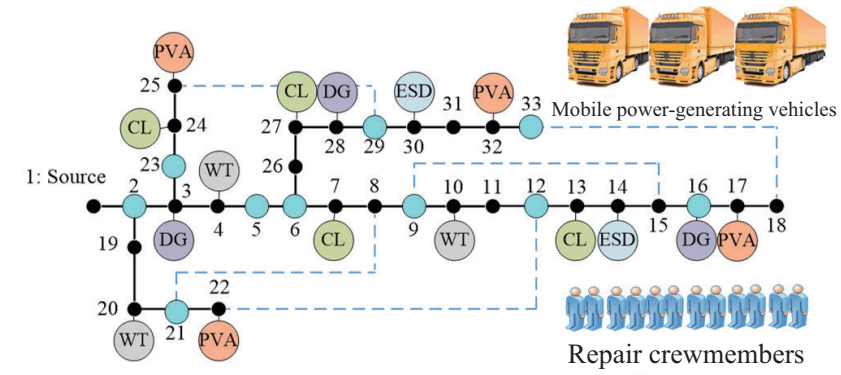

Fig. 5. The test system with integration of resilient resources.

to a modified 33-node distribution system. The system is integrated with various resilient resources including three wind turbines (WTs), four PV arrays (PVAs), two energy storage devices (ESDs), four controllable loads (CLs) and three diesel generators (DGs). Their integrated positions are shown in Fig. 5. Besides, three mobile power-generating vehicles and twelve repair crewmembers are dispatchable if available. The allowable nodes for vehicle connection are marked blue in Fig. 5.

\section{A. Data Preparation}

To validate our proposed method on the test system, which has no historical data, a data generation process is needed before performing the online risk assessment. The risk in hurricane weather is taken as an illustrative example, but the method can be extended to other extreme events. The timeseries load data is from the typical daily load profile [23] of Guangdong province, which suffers several hurricanes every year. The data is then scaled to fit the magnitude in the 33-node system. The wind speed data during hurricane weather are provided by Guangzhou Power Supply Bureau. The generation principles of the historical time-series data in Table I are as follows:

1) Generating short-term reliability data (index No. 1-4). The short-term reliability data can be obtained by the failuremode-and-effect-analysis (FMEA) [4], and this paper considers N-1, N-2 and N-3 branch failure scenarios, where the failure rate and repair time of branches are affected by real-time wind speed [24]. For simplicity, the failure rate and repair time are assumed to have the following relationships with wind speed:

$$
\begin{aligned}
& \lambda_{t}(v)=\left\{\begin{array}{l}
\lambda_{n}, v \leqslant v_{\mathrm{cr} 1} \\
\frac{\left(v^{2}-v_{\mathrm{cr} 1}^{2}\right)-\lambda_{n}\left(v^{2}-v_{\mathrm{cr} 2}^{2}\right)}{v_{\mathrm{cr} 2}^{2}-v_{\mathrm{cr} 1}^{2}}, v_{\mathrm{cr} 1}<v<v_{\mathrm{cr} 2}, \\
1, v \geqslant v_{\mathrm{cr} 2}
\end{array}\right. \\
& r_{t}(v)=\left\{\begin{array}{l}
r_{n}, v \leqslant v_{\mathrm{cr} 3} \\
\beta\left(v-v_{\mathrm{cr} 3}\right)+r_{\mathrm{n}}, v>v_{\mathrm{cr} 3},
\end{array}\right.
\end{aligned}
$$

where $\lambda_{t}$ and $r_{t}$ are the failure rate and repair time in hurricane weather, $\lambda_{\mathrm{n}}$ and $r_{\mathrm{n}}$ are the parameters in normal condition, $v_{\mathrm{crl}}$, $v_{\mathrm{cr} 2}$ and $v_{\mathrm{cr} 3}$ are the thresholds.

2) Generating operation and resource data (index No. 5-9 


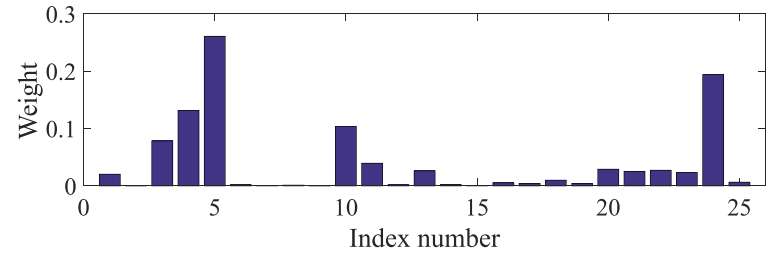

Fig. 6. The weights of indexes determined by the entropy method.

and No. 12-23). Perform a sequential sampling of branch states (normal or failure), and do the FEMA to obtain the grid operational data of index No. 6-9. The data of index 23 can also be easily obtained. The data of resilient resources of index No. 12, 14, 16 and 18 are decided by the availabilities of facility, which are supposed to follow the Bernoulli distributions. The initial data of index No. 13, 15, 17 and 19 are randomly generated when an outage scenario occurs, and the subsequent data as well as the data of index No. 5 can obtained with a rough dispatch simulation of distribution system resources. The other data of index No. 20-22 are supposed to follow the uniform distributions at their respective ranges.

3) Weather-related data (index No. 10-11 and 24-25). The renewable output forecast data (index No. 10-11) are obtained by transforming the wind speed and solar irradiance data into the power data, using the characteristic functions in [25]. The meteorology data (index No. 24 and 25) of historical hurricanes are from the website of Guangzhou Meteorological Service [26].

\section{B. Model Training and Parameter Selection}

The procedures of model training include: 1) determining the weights of indexes; 2) risk quantification by gray correlation degree; and 3) parameter selection in the GCA model.

First, the weights of the 25 basic indexes are determined by the entropy weight method. The result is shown in Fig. 6. It can be learned from Fig. 6 that, index No. 5 (ratio of current loss of load) can provide most useful information for decision making, followed by index No. 24, 4, 10 and 3, indicating the significance of load and meteorology data in online risk assessment. The resilient and emergency resources also contribute a lot to the risk.

Then, the gray correlation degrees for all historical samples are calculated with a pre-given resolution coefficient $\rho$. Two kinds of risk maps are chosen for different risk references of users, and their definitions are:

$$
\begin{aligned}
\operatorname{risk}_{1}(t)= & \left(G(t)-G_{\text {min }}+R_{\text {min }}\right)\left(R_{\text {max }}-R_{\text {min }}\right) /\left(G_{\text {max }}-G_{\text {min }}\right) \\
& \operatorname{risk}_{2}(t)=\left[\left(G(t)-\mu_{\mathrm{G}}\right) / \sigma_{\mathrm{G}}\right] \cdot \sigma_{\mathrm{R}}+\mu_{\mathrm{R}}
\end{aligned}
$$

where $G(t)$ is the gray correlation degree time-series; $G_{\min }$, $G_{\max }, R_{\min }$ and $R_{\max }$ are the minimum and maximum values of gray correlation degree and resilience metric time-series; $\mu_{\mathrm{G}}$, $\sigma_{\mathrm{G}}, \mu_{\mathrm{R}}$ and $\sigma_{\mathrm{R}}$ are the means and standard deviations of gray correlation degree and resilience metric time-series.

The historical risk time-series calculated with (14) and (15), as well as the resilience metric time-series, are shown in Fig. 7.

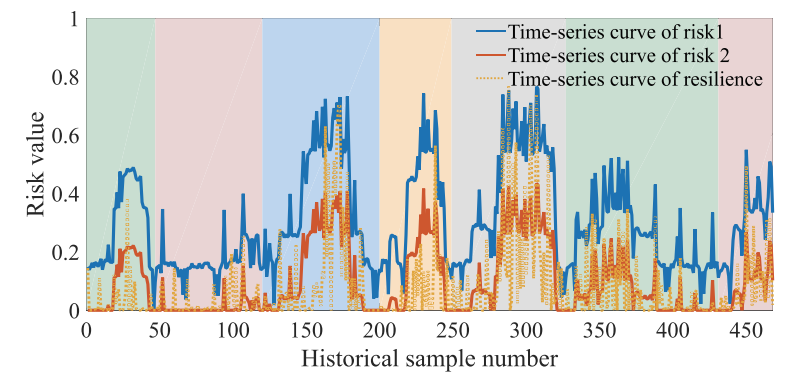

Fig. 7. Historical risk curves in different risk references.

TABLE IV

ERROR INFORMATION FOR TwO RISK MAPS

\begin{tabular}{lcccc}
\hline \hline No. & $E_{1}$ & $E_{2}$ & $E_{3}$ & $E_{4}$ \\
\hline 1 & 0.2251 & 0.2244 & 0.0007 & 0.6340 \\
2 & 0.0690 & 0.0441 & 0.0249 & 0.6569 \\
\hline \hline
\end{tabular}

The hurricanes are indicated with different background colors in the figure. It can be learned that the third, fourth, and fifth hurricanes (2017-13 Hato, 2017-14 Pakhar and 2017-20 Khanun) have higher average risks than the other hurricanes, which is mainly due to the high hurricane intensity, but is also related to some other factors like resilient resources and emergency response, which will be explained in Section D.

Another interesting find in Fig. 7 is that, both risk 1 and 2 can reflect the real risk (quantified by resilience metric) to some extent, but they have differences. We define four indexes to characterize the error between the risk and resilience curve:

$$
E_{1}=\frac{1}{m} \sum_{i=1}^{m}|\operatorname{risk}(i)-\operatorname{resilience}(i)|,
$$

$E_{2}=\frac{1}{m} \sum_{i=1}^{m}[\operatorname{risk}(i)-\operatorname{resilience}(i)], \operatorname{risk}(i)>\operatorname{resilience}(i)$,

$E_{3}=\frac{1}{m} \sum_{i=1}^{m}[\operatorname{resilience}(i)-\operatorname{risk}(i)], \operatorname{risk}(i)<\operatorname{resilience}(i)$,

$$
E_{4}=\rho(\text { risk, resilience }) \text {, }
$$

where $m$ is number of historical samples; and $\rho$ is the correlation coefficient. Therefore, $E_{1}$ can quantify the approximation degree between two curves; $E_{2}$ and $E_{3}$ can quantify the over- and underestimate probabilities and degrees of specific risk map; $E_{4}$ can quantify the synchronism of fluctuation. The error information for the two risk maps is shown in Table IV.

It can be learnt from Table IV that, the risk curve by map 2 is more approximate to the resilience curve, but it has relatively large probability of risk under-estimation. By contrast, even the risk curve by map 1 has a certain degree of deviation from the resilience curve, its variation trend is consistent with the real risk, and the probability of under-estimation is very small. For a safe reason, the risk map 1 is chosen for the rest of the study.

Finally, the parameter $\rho$ in GCA is determined with sensi- 

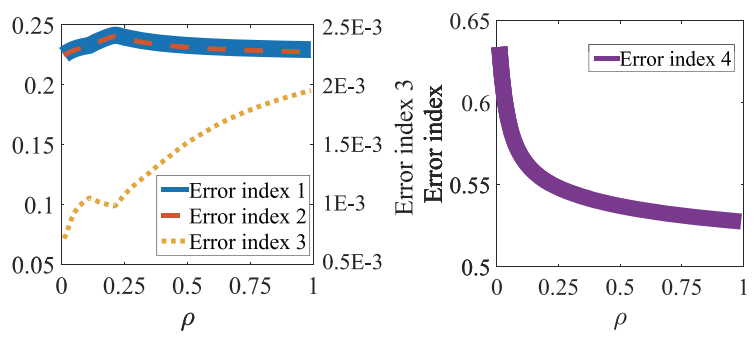

Fig. 8. Sensitivity analysis of different values of parameter $\rho$.

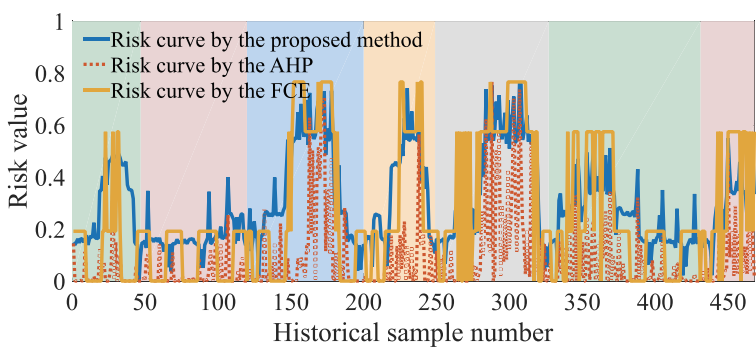

Fig. 9. Risk curves calculated by three different methods.

tivity analysis. Setting the value of $\rho$ from 0 to 1 , the four error indexes are calculated and shown in Fig. 8.

It can be observed from Fig. 8 that with increasing $\rho$, error indexes 1 and 2 first increase and then decrease, index 3 basically increases and index 4 monotonously decreases. The best value of $\rho$ can be therefore found at 0 . Moreover, it can be known that different $\rho$ would cause little error to the risk value (from distance indexes 1, 2 and 3), but would greatly affect the fluctuation trend of time-series risk curve (from correlation index 4). Therefore, the parameter should be carefully selected before applied to online risk assessment.

\section{Methods Comparison and Validation}

To validate the effectiveness of the proposed method, it is compared with the similar approaches AHP and FCE mentioned in the introduction section. The pairwise comparison matrix of the 25 risk indexes in AHP is constructed by expert system, and can be found in Appendix A. The coincidence indicator $\mathrm{CI}=(25.9218-25) / 24=0.0384$, and the coincidence ratio $\mathrm{CR}=\mathrm{CI} / \mathrm{RI}=0.0384 / 1.6556=0.0232<0.1$, which means the constructed matrix passes the coincidence test and can be used in AHP. The FCE is performed for each historical sample by observing the values of 25 indexes and making a fuzzy judge of the risk level it could belong to. The judging standard is also decided by expert system and presented in Appendix B. The risk curves by the three methods are shown in Fig. 9, and their errors with the resilience curves are listed in Table V.

It can be learnt from Table $\mathrm{V}$ that, the risk curves by the three methods have similar deviations from the ideal resilience curve, but the proposed method has very low probability of risk under-estimation (0.0007), compared with the AHP (0.0092) and FCE (0.0111). Besides, the high correlation coefficient of the proposed method also indicates it has better performance in
TABLE V

ERROR INFORMATION FOR DiFFERENT AsSESSMENT METHODS

\begin{tabular}{lcccc}
\hline \hline \multicolumn{1}{c}{ Name } & $E_{1}$ & $E_{2}$ & $E_{3}$ & $E_{4}$ \\
\hline Proposed & 0.2251 & 0.2244 & 0.0007 & 0.6340 \\
AHP & 0.2183 & 0.2091 & 0.0092 & 0.4312 \\
FCE & 0.2059 & 0.1948 & 0.0111 & 0.4940 \\
\hline \hline
\end{tabular}

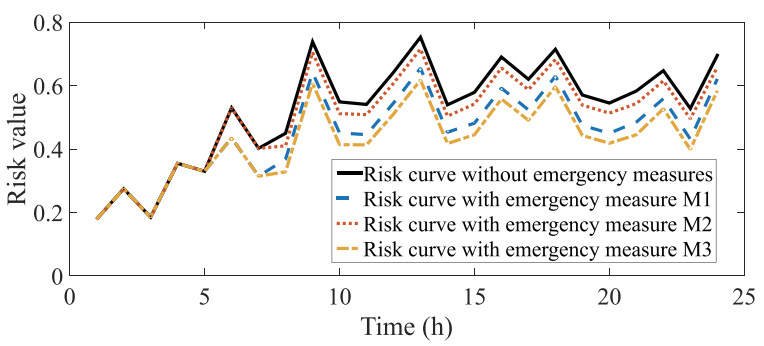

Fig. 10. Risk curves for the next 24 hours with different emergency measures.

TABLE VI

Overall Risk Metric Values With Different Measures

\begin{tabular}{lcccc}
\hline & No measures & M1 & M2 & M3 \\
\hline Area & 0.5269 & 0.4535 & 0.5023 & 0.4289 \\
Degree & 0.7530 & 0.6551 & 0.7157 & 0.6177 \\
Time & 0.3333 & 0.1667 & 0.2917 & 0.0833 \\
\hline \hline
\end{tabular}

characterizing the variation trend of system risk than the AHP and FCE. Therefore, the proposed method is more reliable in the real application of online risk assessment.

\section{Application to Online Risk Assessment}

A real-time instant is taken as an example for online risk assessment. The time-series data for the next 24 hours can be forecasted in some way for some indexes such as the reliability (index No. 1-4), grid (index No. 6-9), renewable (index No. 10-11), and meteorological indexes (index No. 24-25), while the other data can be assumed to be constant or roughly estimated. To enhance the system resilience, three emergent measures are carried out, including: (M1) increasing emergency power sources and re-inforcement of repair crew and supplies at the sixth hour (index No. 18-22 set to 1), (M2) increasing resilient resources at the eighth hour (index No. 1217 set to 1), and (M3) combination of M1 and M2. The risk assessment results are shown in Fig. 10, and three risk metrices are defined as follows for the next 24 hours. Their values for different measures are listed in Table VI.

$$
\begin{gathered}
\text { Area }=\left(\sum_{i=1}^{24} \operatorname{risk}(i)\right) / 24 \\
\text { Degree }=\max _{1 \leqslant i \leqslant 24} \operatorname{risk}(i) \\
\text { Time }=\underset{1 \leqslant i \leqslant 24}{N}\left[\operatorname{risk}(i)>R_{\mathrm{set}}\right] / 24
\end{gathered}
$$

where $N\{\bullet\}$ is the number of time instants that satisfy the 
condition in the brace; and $R_{\text {set }}$ is the threshold and is set as 0.6 .

It can be known from Fig. 10 and Table VI that, by carrying out emergency measures, the system resilience can be significantly improved. In this test system, increasing emergency power sources and reinforcement of repair crew and supplies (M1) performs better than increasing resilient resources (M2), and the combination of M1 and M2 (M3) performs the best. However, even in the circumstance of M3, the system still has an average risk of 0.4289 for the next 24 hours, which means the resilience of the system is still not enough, and more resilient resources should be put into it, and the capabilities of emergency response and repair should be further enhanced.

\section{CONCLUSION}

This paper proposes a novel data-driven online risk assessment method for resilient distribution systems suffering from extreme events. The key findings can be concluded as follows:

1) The proposed combined entropy weight and gray correlation degree method performs better than the similar approaches AHP and FCE in online risk assessment.

2) The factors of resilient resources and emergency response would affect the accuracy of risk assessment results.

3) The proposed online risk assessment scheme can help make decision for improving distribution system resilience.

In our future work, the optimization of various resilient resources will be combined with the proposed risk assessment procedures to construct a more resilient distribution system.

\section{APPENDIX A \\ Paiwise Comparison Matrix IN THE AHP}

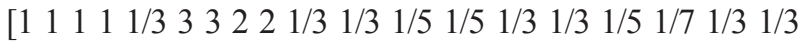
$1 / 31 / 3 \quad 1 / 33 \quad 1 / 7$ 1/7; $1111111 / 3332221 / 31 / 31 / 51 / 51 / 31 / 3$

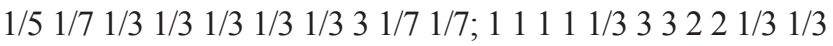
$1 / 5 \quad 1 / 5 \quad 1 / 3 \quad 1 / 3 \quad 1 / 5 \quad 1 / 7 \quad 1 / 3 \quad 1 / 3 \quad 1 / 3 \quad 1 / 3 \quad 1 / 3 \quad 3 \quad 1 / 7$ 1/7; $111111 / 3$

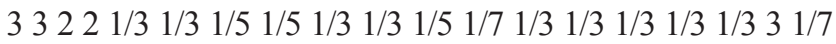
1/7; $333315544111 / 31 / 311$ 1/3 1/5 $111111^{1 / 5}$ 1/5;

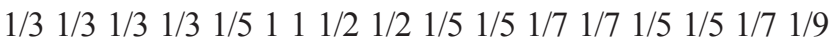

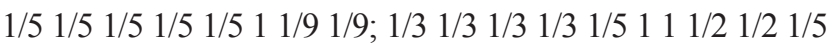

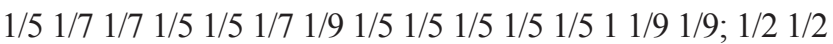

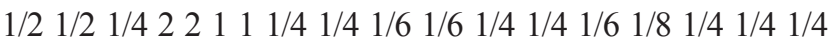

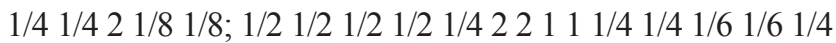
$1 / 41 / 61 / 81 / 41 / 41 / 41 / 41 / 421 / 81 / 8 ; 33331554411$ $1 / 31 / 3111 / 31 / 51111151 / 51 / 5 ; 333315544111 / 3$ $1 / 3111 / 31 / 51111151 / 51 / 5 ; 555537766331133$ $11 / 33333371 / 31 / 3 ; 55553776633113311 / 333$ $33371 / 31 / 3$; $333315544111 / 31 / 3111 / 31 / 51111$ $151 / 51 / 5 ; 333315544111 / 31 / 3111 / 31 / 5111115$ $1 / 51 / 5 ; 55553776633113311 / 33333371 / 31 / 3 ; 7$ $777599885533553155555911 ; 333315544$ $111 / 31 / 3111 / 31 / 51111151 / 51 / 5 ; 33331554411$ $1 / 31 / 3111 / 31 / 51111151 / 51 / 5 ; 333315544111 / 3$ $1 / 3111 / 31 / 51111151 / 51 / 5 ; 333315544111 / 31 / 3$ $111 / 31 / 51111151 / 51 / 5 ; 333315544111 / 31 / 311$

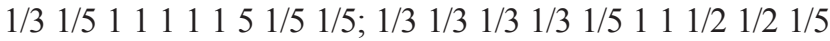

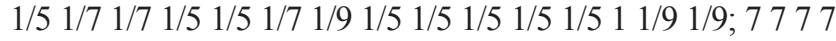
$599885533553155555911 ; 777759988553$ 3553155555911 ].

\section{APPENDIX B}

Fuzzy Judgement Standard of Risk LeVEl in the FCE

The risk range is divided into 5 levels uniformly: $[0,0.2]$, $[0.2,0.4],[0.4,0.6],[0.6,0.8]$ and $[0.81 .0]$, and the median in each interval is taken as the risk value of the corresponding level. The weight vector for index No. 1-5, 6-9, 10-22, 23 and $24-25$ is [6/21 2/21 4/21 1/21 8/21], and the membership functions are [ $\left.\begin{array}{llllll}0.3 & 0.4 & 0.2 & 0.1 & 0\end{array}\right],\left[\begin{array}{lllll}0.3 & 0.3 & 0.3 & 0.1 & 0\end{array}\right]$, [ $\begin{array}{lllll}0.3 & 0.3 & 0.2\end{array}$ $\left.\begin{array}{ll}0.1 & 0.1\end{array}\right],\left[\begin{array}{lllll}0.25 & 0.25 & 0.2 & 0.15 & 0.15\end{array}\right]$, and [ $\left.\begin{array}{lllll}0.4 & 0.3 & 0.2 & 0.1 & 0\end{array}\right]$ for the 5 risk levels if their index values are better than the average. Otherwise, the corresponding membership function is flipped.

\section{REFERENCES}

[1] W. Li, Risk Assessment of Power Systems. New York: IEEE, 2014.

[2] Z. Bie, P. Zhang, G. Li, B. Hua, M. Meehan, and X. Wang, "Reliability evaluation of active distribution systems including microgrids," in IEEE Transactions on Power Systems, vol. 27, no. 4, pp. 2342-2350, Nov. 2012.

[3] R. Billinton and P. Wang, "Teaching distribution system reliability evaluation using Monte Carlo simulation," in IEEE Transactions on Power Systems, vol. 14, no. 2, pp. 397-403, May 1999.

[4] R. Billinton and P. Wang, "Reliability-network-equivalent approach to distribution-system-reliability evaluation," in Proceedings of IEE Proceedings - Generation, Transmission and Distribution, vol. 145, no. 2, pp. 149-153, Mar. 1998.

[5] S. R. K. Yeddanapudi, Y. Li, J. D. McCalley, A. A. Chowdhury, and W. T. Jewell, "Risk-based allocation of distribution system mainten-ance resource," in IEEE Transactions on Power Systems, vol. 23, no. 2, pp. 287-295, May 2008

[6] R. Billinton and Z. Pan, "Historic performance-based distribution system risk assessment," in IEEE Transactions on Power Delivery, vol. 19, no. 4, pp.1759-1765, Oct. 2004.

[7] E. Akhavan-Rezai, M. -R. Haghifam, and A. Fereidunian, "Data-driven reliability modeling, based on data mining in distribution network fault statistics," in Proceedings of 2009 IEEE Bucharest Power Tech, 2009, pp. $1-6$.

[8] L. Hu, K. Liu, Y. Diao, X. Meng, and W. Sheng, "Operational reliability evaluation method based on big data technology," in Proceedings of 2016 International Conference on Cyber-Enabled Distributed Computing and Knowledge Discovery (CyberC), Chengdu, China, 2016, pp. 341-344.

[9] X. Chen, J. Tang, Q. Chang, and W. Li, "A data-driven method for operational reliability prediction on electric devices considering multiple meteorological factors," in Proceedings of 2018 IEEE International Conference on Probabilistic Methods Applied to Power Systems (PMAPS), Boise, ID, USA, 2018, pp. 1-6.

[10] Z. Ruifeng, H. Shuqing, F. Yu, W. Zhuoyue, and W. Yi, "Research on situation evaluation and prediction method for distribution network," in 2020 4th International Conference on Power and Energy Engineering (ICPEE), 2020, pp. 7-12.

[11] Z. Xin, L. Chen, Y. Yang, K. Miao, and Z. Li, "Application of AHP-DEA model in comprehensive evaluation of distribution network planning reliability," in 2019 IEEE Sustainable Power and Energy Conference (iSPEC), 2019, pp. 290-294.

[12] V. Naseri, B. Kashanizadeh, and F. Varposhti, "Online operational budgeting of electricity distribution network based on integration strategic and operational planning by Swot \& AHP methods," in 2018 Electrical Power Distribution Conference (EPDC), 2018, pp. 63-68.

[13] L. Ma, W. Liu, and T. -m. Zhu, "Methods selection and overall process of distribution network planning comprehensive evaluation," in 2014 China International Conference on Electricity Distribution (CICED), 2014, pp. 633-636.

[14] Liming Duan, Nian Liu, Zongqi Liu, and Jianhua Zhang, "A 
comprehensive evaluation method for distribution networks'disaster vulnerability based on group AHP," in 2012 China International Conference on Electricity Distribution, 2012, pp. 1-5.

[15] Y. F. Wang, M. H. Deng, Y. K. Bao, H. Zhang, J. Y. Chen, J. Qian, and C. X. Guo, "Power system disaster-mitigation dispatch platform based on big data," in Proceedings of 2014 International Conference on Power System Technology, Chengdu, China, 2014, pp. 1014-1019.

[16] E. Ciapessoni, D. Cirio, M. Lacavalla, M. De Nigris, A. Pitto, and M. Sforna, "Risk-based security assessment with big data driven probabilistic modeling for wet snow extreme events," in Proceedings of 2018 Power Systems Computation Conference (PSCC), Dublin, Ireland, 2018, pp. 1-7.

[17] X. Li, L. Zhang, F. Liu, Y. Liang, C. Lin, H. Tian, G. Li, and Z. Bie, "Data-driven real-time risk assessment of resilient distribution system during typhoon weather," in Proceedings of 2020 4th International Conference on HVDC (HVDC), Xi'an, China, 2020, pp. 786-791.

[18] International Strategy for Disaster Reduction, "Living with risk: a global review of disaster reduction initiatives," United Nations, Geneva, Switzerland, 2004.

[19] M. Panteli and P. Mancarella, "The grid: stronger, bigger, smarter?: Presenting a conceptual framework of power system resilience," in IEEE Power and Energy Magazine, vol. 13, no. 3, pp. 58-66, May-Jun. 2015.

[20] J. Xiong, J. Li, and Y. Yang, "Method of determine index weight in security risk evaluation based on information entropy," in Proceedings of 2012 Fourth International Conference on Multimedia Information Networking and Security, Nanjing, China, 2012, pp. 43-48.

[21] W. Li, Y. Shang, and Y Ji, "Analysis of multiple objective decision methods based on entropy weight," in Proceedings of 2008 IEEE PacificAsia Workshop on Computational Intelligence and Industrial Application, Wuhan, China, 2008, pp. 953-956.

[22] M. Yang, M. Deng, R. Jia, and Y. Liu, "Research on index weight based on improved grey relational analysis," in Proceedings of 2010 International Conference on Machine Learning and Cybernetics, Qingdao, China, 2010, pp. 1967-1970.

[23] J. Huan and Z. Zuo, "Research on typical day selection method of Guangdong power grid load characteristics," in Journal of Electric Power ence and Technology(in Chinese), vol. 32, no. 1, pp. 164-170, Mar. 2017.

[24] G. Li, P. Zhang, P. B. Luh, W. Li, Z. Bie, C. Serna, and Z. Zhao, "Risk analysis for distribution systems in the northeast U.S. under wind storms," in IEEE Transactions on Power Systems, vol. 29, no. 2, pp. 889-898, Mar. 2014.

[25] K. Zou, A. P. Agalgaonkar, K. M. Muttaqi, S. Perera, and N. Browne, "Support of distribution system using distributed wind and PV systems," in Proceedings of 2009 Australasian Universities Power Engineering Conference, Adelaide, SA, Australia, 2009, pp. 1-5.

[26] Guangzhou Meteorology, "Historical data inquiry of weather alarm," [online]. Available: http://www.tqyb.com.cn/gz/weatherAlarm/history/.

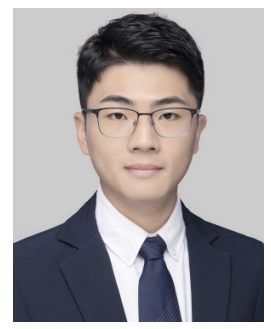

Chaofan Lin received the B.S. degree in electrical engineering from Xi'an Jiaotong University, Xi'an, China, in 2017.

$\mathrm{He}$ is currently pursuing the Ph.D. degree in the State Key Laboratory of Electrical Insulation and Power Equipment (Institute of Power System and Its Resilience, Xi'an Jiaotong University). His research interest is power system risk and resilience assessment with renewable energy integration.

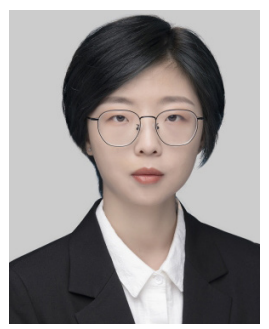

Fei Liu received the B.S. degree in electrical engineering from Xi'an Jiaotong University, Xi'an, China, in 2020.

She is currently pursuing the master degree in the State Key Laboratory of Electrical Insulation and Power Equipment (Institute of Power System and Its Resilience, Xi'an Jiaotong University). Her research interests include power system resilience, and power system simulation and control.

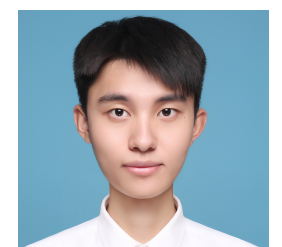

Liyin Zhang received the B.S. degree in electrical engineering from Xi'an Jiaotong University, Xi'an, China, in 2020 .

$\mathrm{He}$ is currently pursuing the $\mathrm{Ph} . \mathrm{D}$. degree in the State Key Laboratory of Electrical Insulation and Power Equipment (Institute of Power System and Its Resilience, Xi'an Jiaotong University). His research interests include resilient power system and machine learning.

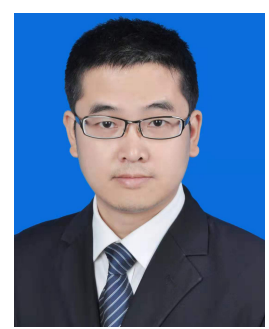

Gengfeng $\mathbf{L i}$ received the Ph.D. degree in electrical engineering from Xi'an Jiaotong University, Xi'an, China, in 2014.

$\mathrm{He}$ is currently an associate professor with the State Key Laboratory of Electrical Insulation and Power Equipment (Institute of Power System and Its Resilience, Xi'an Jiaotong University). His research interests include power system reliability evaluation and integration of renewable energy.

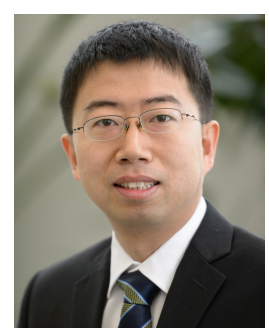

Chen Chen received the B.S. and M.S. degrees from Xi'an Jiaotong University, Xi'an, China, in 2006 and 2009 , respectively, and the $\mathrm{Ph} . \mathrm{D}$. degree in electrical engineering from Lehigh University, Bethlehem, PA, USA, in 2013.

$\mathrm{He}$ is currently a professor with the State Key Laboratory of Electrical Insulation and Power Equipment (Institute of Power System and Its Resilience, Xi'an Jiaotong University). His research interests include power system resilience, distribution systems and microgrids, demandside management, and communications and signal processing for smart grid.

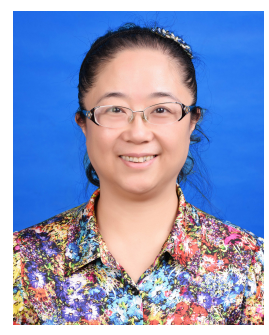

Zhaohong Bie received the B.S. and M.S. degrees from Shandong University, Jinan, China, in 1992 and 1994, respectively, and the Ph.D. degree from Xi'an Jiaotong University, Xi'an, China, in 1998.

She is currently a professor with the State Key Laboratory of Electrical Insulation and Power Equipment (Institute of Power System and Its Resilience, Xi'an Jiaotong University). Her research interests include power system planning and reliability evaluation, as well as renewable energy integration. 